\title{
INCREASED LEVELS OF BISPHENOL A IN EUTHYROID AUTOIMMUN THYROIDITIS
}

\section{Esra Ademoglu ${ }^{1}$, Muge Keskin ${ }^{2}$, Suheyla Gorar ${ }^{3}$, Ayse Carloglu ${ }^{4}$}

${ }^{1}$ Bolu Abant Izzet Baysal University Hospital, Department of Endocrinology and Metabolism

${ }^{2}$ Ankara Training and Education Hospital, Department of Endocrinology and Metabolism

${ }^{3}$ Antalya Training and Educatşon Hospital, Department of Endocrinology and Metabolism

${ }^{4}$ Erzurum Training and Education Hospital, Department of Endocrinology and Metabolism

\section{Objectives:}

Autoimmune thyroiditis (AIT), also called Hashimoto thyroiditis or chronic lymphocytic thyroiditis is characterized by destruction of the thyroid gland involving apoptosis of thyroid epithelial cells. It usually presents as euthyroidism or subclinically hypothyroidism, rarely as overt hypothyroidism. The cause of Hashimoto's thyroiditis is thought to be a combination of genetic susceptibility and environmental factors. Bisphenol A (BPA), an endocrine-disrupting chemical, is the main component of polycarbonate plastics and is one of the highest volume chemicals in production today. The human population is widely and continuously exposed to BPA through food, drinking water, dermal exposures, and inhalation of dusts. The aim of the study is to evaluate circulating levels of BPA in euthyroid AIT.

Circulating levels of BPA were measured in 20 consecutive patients with newly diagnosed euthyroid AIT and 22 euthyroid controls. Patients were considered to have AIT if they were positive for at least one of either AbTPO or AbTg, in addition to exhibiting morphological changes consistent with AIT, as diagnosed by thyroid ultrasound (hypoechogenicity, heterogeneity). Individuals were classified as euthyroid AIT at diagnosis if they had normal serum TSH $(\mathrm{TSH}<4 \mathrm{mIU} / \mathrm{mL})$ and normal free T4 concentrations.

Graphs and tables

Table-1.Biochemical values in euthyroid AIT and controls.

\begin{tabular}{lll}
\hline Parameters & Euthyroid AlT & \multicolumn{1}{c}{ Controls } \\
& & \\
\hline BPA & $0.94 \pm 0.85^{\star}$ & $0.46 \pm 0.54^{\star}$ \\
TSH & $2.20 \pm 1.23$ & $1.71 \pm 0.69$ \\
fT4 & $1.09 \pm 0.10$ & $1.11 \pm 0.13$ \\
fT3 & $3.07 \pm 0.28$ & $3.14 \pm 0.25$ \\
Anti-TPO & $1300.0 \pm 0.0^{\star}$ & $52.30 \pm 15.27^{\star}$ \\
Anti-Tg & $217.38 \pm 142.58^{\star}$ & $26.27 \pm 11.18^{\star}$
\end{tabular}

BPA, Bisphenol-A; TSH, thyroid stimulating hormone; fT4, free thyroxine; fT3, free triiodothyronine; Anti-TPO, anti thyroid peroxidase; Anti-Tg, anti-thyroglobulin. ${ }^{*} \mathrm{p}<0.05$, statistically significant. NS, statistically nonsignificant.
Table-2. The correlation analyses of bisphenol-A with some parameters in euthyroid AIT and controls.

\begin{tabular}{lll}
\hline Parameters & r value & p value \\
\hline Anti-TPO & 0.355 & \\
Anti-Tg & 0.099 & $0.027^{\star}$ \\
TSH & 0.046 & 0.550 \\
fT & 0.027 & 0.781 \\
fT3 & 0.102 & 0.870 \\
& & 0.537
\end{tabular}

Anti-TPO, anti thyroid peroxidase; Anti-Tg, anti-thyroglobuin; TSH, thyroid stimulating hormone;fT4, free thyroxine; $\mathrm{fT} 3$, free triiodothyronine. ${ }^{\star} \mathrm{p}<0.05$ is statistically significant.

\section{Results:}

Circulating levels of bisphenol a was significantly higher in euthyroid AIT compared with controls $(p<0.05)$. In correlation analysis, bisphenol a was positively correlated with anti TPO $(r=0.355, p<0.05)$.

\section{Conclusions:}

The present study demonstrated for the first time that circulating bisphenol a levels are increased in euthyroid autoimmune thyroiditis. This result draws attention to the circulating levels of bisphenol a in euthyroid autoimmune thyroiditis and and may shed light on further researches at this topic. 